CUBO A Mathematical Journal

Vol.15, № 02, (01-19). June 2013

\title{
Uniform Convergence With Rates of General Singular Operators
}

\author{
George A. Anastassiou and Razvan A. Mezei \\ The University of Memphis \\ Department of Mathematical Sciences, \\ Memphis, TN 38152, U.S.A. \\ ganastss@memphis.edu, rmezei@memphis.edu
}

\begin{abstract}
In this article we study the approximation properties of general singular integral operators over the real line. We establish their convergence to the unit operator with rates. The estimates are mostly sharp and they are pointwise or uniform. The established inequalities involve the higher order modulus of smoothness. We apply this theory to the trigonometric singular operators.
\end{abstract}

\section{RESUMEN}

En este artículo estudiamos propiedades de aproximación de operadores integrales singulares generales sobre la recta real. Establecemos su convergencia al operador unidad con las tasas correspondientes. Las estimaciones son mayormente ajustadas y son tanto puntuales como uniformes. Las desigualdades encontradas involucran el módulo de suavidad de alto orden. Aplicamos esta teoría a los operadores singulares trigonométricos.

Keywords and Phrases: Best constant, general singular integral, trigonometric singular integral, modulus of smoothness, sharp inequality.

2010 AMS Mathematics Subject Classification: 26A15, 41A17, 41A35. 


\section{Introduction}

The rate of convergence of singular integrals has been studied earlier in [5], 12], [13, [15, 3], [6] [7, [8], [9] and these articles motivate this work. Here we consider some very general singular integral operators over $\mathbb{R}$ and we study the degree of approximation to the unit operator with rates over smooth functions. We establish related inequalities involving the higher modulus of smoothness with respect to $\|\cdot\|_{\infty}$. The estimates are pointwise and uniform. Most of the times these are optimal in the sense that the inequalities are attained by basic functions. We give particular applications of these operators to the trigonometric singular integral operators over $\mathbb{R}$. The discussed operators are not in general positive.

Other motivation comes from [1], 2].

\section{Main Results}

In the next we study the following smooth general singular integral operators $\Theta_{r, \xi}(f, x)$ defined as follows. Let $\xi>0$ and let $\mu_{\xi}$ be Borel probability measures on $\mathbb{R}$.

For $r \in \mathbb{N}$ and $n \in \mathbb{Z}_{+}$we put

$$
\alpha_{j}= \begin{cases}(-1)^{r-j}\left(\begin{array}{l}
r \\
j
\end{array}\right) j^{-n}, & j=1, \ldots, r, \\
1-\sum_{j=1}^{r}(-1)^{r-j}\left(\begin{array}{l}
r \\
j
\end{array}\right) j^{-n}, & j=0,\end{cases}
$$

that is $\sum_{j=0}^{r} \alpha_{j}=1$. Let $f: \mathbb{R} \rightarrow \mathbb{R}$ be Borel measurable, we define for $x \in \mathbb{R}$, the integral

$$
\Theta_{r, \xi}(f ; x):=\int_{-\infty}^{\infty}\left(\sum_{j=0}^{r} \alpha_{j} f(x+j t)\right) d \mu_{\xi}(t) .
$$

We suppose that $\Theta_{r, \xi}(f ; x) \in \mathbb{R}$ for all $x \in \mathbb{R}$. We will use also that

$$
\Theta_{r, \xi}(f ; x)=\sum_{j=0}^{r} \alpha_{j}\left(\int_{-\infty}^{\infty} f(x+j t) d \mu_{\xi}(t)\right) .
$$

We notice that $\Theta_{r, \xi}(\mathbf{c}, x)=\mathbf{c}, \mathbf{c}$ constant, and

$$
\Theta_{r, \xi}(f ; x)-f(x)=\sum_{j=0}^{r} \alpha_{j}\left(\int_{-\infty}^{\infty} f(x+j t)-f(x)\right) d \mu_{\xi}(t) .
$$

Let $f \in C^{n}(\mathbb{R}), n \in \mathbb{Z}^{+}$with the rth modulus of smoothness finite, i.e.

$$
\omega_{r}\left(f^{(\mathfrak{n})}, h\right):=\sup _{|t| \leq h}\left\|\Delta_{\mathrm{t}}^{\mathrm{r}} f^{(\mathfrak{n})}(\mathrm{x})\right\|_{\infty, x}<\infty, \quad h>0
$$


where

$$
\Delta_{t}^{r} f^{(n)}(x):=\sum_{j=0}^{r}(-1)^{r-j}\left(\begin{array}{l}
r \\
j
\end{array}\right) f^{(n)}(x+j t)
$$

see [10, p. 44.

We need to introduce

$$
\delta_{k}:=\sum_{j=1}^{r} \alpha_{j} j^{k}, \quad k=1, \ldots, n \in \mathbb{N},
$$

and the even function

$$
G_{n}(t):=\int_{0}^{|t|} \frac{(|t|-w)^{n-1}}{(n-1) !} \omega_{r}\left(f^{(n)}, w\right) d w, \quad n \in \mathbb{N}
$$

with

$$
\mathrm{G}_{0}(\mathrm{t}):=\omega_{\mathrm{r}}(\mathrm{f},|\mathrm{t}|), \quad \mathrm{t} \in \mathbb{R}
$$

Denote by $\lfloor\cdot\rfloor$ the integral part.

We present our first result

Theorem 1. The integrals $c_{k, \xi}:=\int_{-\infty}^{\infty} t^{k} d \mu_{\xi}(t), k=1, \ldots, n$, are assumed to be finite. Then

$$
\left|\Theta_{r, \xi}(f ; x)-f(x)-\sum_{k=1}^{n} \frac{f^{(k)}(x)}{k !} \delta_{k} c_{k, \xi}\right| \leq \int_{-\infty}^{\infty} G_{n}(t) d \mu_{\xi}(t) .
$$

Proof. By Taylor's formula we obtain

$$
\begin{aligned}
f(x+j t) & =\sum_{k=0}^{n-1} \frac{f^{(k)}(x)}{k !}(j t)^{k}+\int_{0}^{j t} \frac{(j t-z)^{n-1}}{(n-1) !} f^{(n)}(x+z) d z \\
& =\sum_{k=0}^{n-1} \frac{f^{(k)}(x)}{k !}(j t)^{k}+j^{n} \int_{0}^{t} \frac{(t-w)^{n-1}}{(n-1) !} f^{(n)}(x+j w) d w .
\end{aligned}
$$

Multiplying both sides of (11) by $\alpha_{j}$ and summing up we get

$$
\sum_{j=0}^{r} \alpha_{j}(f(x+j t)-f(x))=\sum_{k=1}^{n} \frac{f^{(k)}(x)}{k !} \delta_{k} t^{k}+\mathcal{R}_{n}(0, t)
$$

where

$$
\mathcal{R}_{\mathrm{n}}(0, \mathrm{t}):=\int_{0}^{\mathrm{t}} \frac{(\mathrm{t}-w)^{\mathrm{n}-1}}{(\mathrm{n}-1) !} \tau(w) \mathrm{d} w
$$

with

$$
\tau(w):=\sum_{j=0}^{r} \alpha_{j} j^{n} f^{(n)}(x+j w)-\delta_{n} f^{(n)}(x)
$$


Notice also that

$$
-\sum_{j=1}^{r}(-1)^{r-j}\left(\begin{array}{l}
r \\
j
\end{array}\right)=(-1)^{r}\left(\begin{array}{l}
r \\
0
\end{array}\right)
$$

According to [3], p. 306, [1], we get

$$
\tau(w)=\Delta_{w}^{r} f^{(n)}(x)
$$

Therefore

$$
|\tau(w)| \leq \omega_{r}\left(f^{(n)},|w|\right)
$$

all $w \in \mathbb{R}$ independently of $x$. We do have after integration, see also (44), that

$$
\begin{aligned}
\Theta_{r, \xi}(f ; x)-f(x) & =\int_{-\infty}^{\infty}\left(\sum_{j=0}^{r} \alpha_{j}(f(x+j t)-f(x))\right) d \mu_{\xi}(t) \\
& =\int_{-\infty}^{\infty}\left(\sum_{k=1}^{n} \frac{f^{(k)}(x)}{k !} \delta_{k} t^{k}+\mathcal{R}_{n}(0, t)\right) d \mu_{\xi}(t) \\
& =\sum_{k=1}^{n} \frac{f^{(k)}(x)}{k !} \delta_{k}\left(\int_{-\infty}^{\infty} t^{k} d \mu_{\xi}(t)\right)+\mathcal{R}_{n}^{*}
\end{aligned}
$$

where

$$
\mathcal{R}_{\mathrm{n}}^{*}:=\int_{-\infty}^{\infty} \mathcal{R}_{\mathrm{n}}(0, \mathrm{t}) \mathrm{d} \mu_{\xi}(\mathrm{t}) .
$$

Here by (8) and (13) we get

$$
\left|\mathcal{R}_{\mathrm{n}}(0, \mathrm{t})\right| \leq \int_{0}^{|\mathrm{t}|} \frac{(|\mathrm{t}|-w)^{\mathrm{n}-1}}{(\mathrm{n}-1) !}|\tau(\operatorname{sign}(\mathrm{t}) w)| \mathrm{d} w \leq \mathrm{G}_{\mathrm{n}}(\mathrm{t})
$$

see $[5]$.

Hence by (18) we find

$$
\left|\mathcal{R}_{\mathrm{n}}^{*}\right| \leq \int_{-\infty}^{\infty} \mathrm{G}_{\mathrm{n}}(\mathrm{t}) \mathrm{d} \mu_{\xi}(\mathrm{t})
$$

We also have

$$
\Theta_{r, \xi}(f ; x)-f(x)-\sum_{k=1}^{n} \frac{f^{(k)}(x)}{k !} \delta_{k} c_{k, \xi}=\mathcal{R}_{n}^{*} .
$$

Inequality (10) is now clear via (21) and (20).

Corollary 2. Assume $\omega_{\mathrm{r}}(\mathrm{f}, \xi)<\infty, \xi>0$. Then it holds for $\mathrm{n}=0$ that

$$
\left|\Theta_{r, \xi}(f ; x)-f(x)\right| \leq \int_{-\infty}^{\infty} \omega_{r}(f,|t|) d \mu_{\xi}(t) .
$$


Proof. We observe that

$$
\begin{aligned}
\Theta_{r, \xi}(f ; x)-f(x)= & \int_{-\infty}^{\infty}\left(\sum_{j=1}^{r} \alpha_{j}(f(x+j t)-f(x))\right) d \mu_{\xi}(t) \\
= & \int_{-\infty}^{\infty}\left(\sum_{j=1}^{r}(-1)^{r-j}\left(\begin{array}{l}
r \\
j
\end{array}\right)(f(x+j t)-f(x))\right) d \mu_{\xi}(t) \\
= & \int_{-\infty}^{\infty}\left(\sum_{j=1}^{r}(-1)^{r-j}\left(\begin{array}{l}
r \\
j
\end{array}\right) f(x+j t)\right. \\
& \left.-\left(\sum_{j=1}^{r}(-1)^{r-j}\left(\begin{array}{l}
r \\
j
\end{array}\right)\right) f(x)\right) d \mu_{\xi}(t) \\
\stackrel{14}{=} & \int_{-\infty}^{\infty}\left(\sum_{j=1}^{r}(-1)^{r-j}\left(\begin{array}{l}
r \\
j
\end{array}\right) f(x+j t)\right. \\
& \left.+(-1)^{r}\left(\begin{array}{l}
r \\
0
\end{array}\right) f(x)\right) d \mu \xi(t) \\
= & \int_{-\infty}^{\infty}\left(\sum_{j=0}^{r}(-1)^{r-j}\left(\begin{array}{l}
r \\
j
\end{array}\right) f(x+j t)\right) d \mu_{\xi}(t) \\
\underline{\underline{6}} & \int_{-\infty}^{\infty}\left(\left(\Delta_{t}^{r} f\right)(x)\right) d \mu_{\xi}(t) .
\end{aligned}
$$

I.e. we have proved

$$
\Theta_{r, \xi}(f ; x)-f(x)=\int_{-\infty}^{\infty}\left(\Delta_{t}^{r} f(x)\right) d \mu_{\xi}(t) .
$$

Hence by (23) we derive

$$
\begin{aligned}
\left|\Theta_{r, \xi}(f ; x)-f(x)\right| & \leq \int_{-\infty}^{\infty}\left|\Delta_{t}^{r} f(x)\right| d \mu_{\xi}(t) \\
& \leq \int_{-\infty}^{\infty} \omega_{r}(f,|t|) d \mu_{\xi}(t) .
\end{aligned}
$$

That is proving (22).

Inequality (10) is sharp.

Theorem 3. Inequality (10) at $\mathrm{x}=0$ is attained by $\mathrm{f}(\mathrm{x})=\mathrm{x}^{\mathrm{r}+\mathrm{n}}, \mathrm{r}, \mathrm{n} \in \mathbb{N}$ with $\mathrm{r}+\mathrm{n}$ even.

Proof. As in [3], p. 307, [1, [16], p. 54 and (5), (6) we obtain

$$
\omega_{r}\left(f^{(n)}, t\right)=(r+n)(r+n-1) \cdot \ldots \cdot(r+1) r ! t^{r},
$$


$t>0$. And

$$
G_{n}(t)=r ! t^{r+n}, \quad t \in \mathbb{R} .
$$

Also we have $f^{(k)}(0)=0, k=0,1, \ldots, n$. Thus the right hand side of (10) equals

$$
r ! \int_{-\infty}^{\infty} t^{r+n} d \mu_{\xi}(t) .
$$

The left hand side of (10) equals

$$
\begin{aligned}
& \left|\Theta_{r, \xi}(f ; 0)\right|=\left|\int_{-\infty}^{\infty}\left(\sum_{j=0}^{r} \alpha_{j} f(j t)\right) d \mu_{\xi}(t)\right| \\
& =\left|\int_{-\infty}^{\infty}\left(\sum_{j=1}^{r} \alpha_{j}(j t)^{r+n}\right) d \mu_{\xi}(t)\right| \\
& =\left|\int_{-\infty}^{\infty}\left(\sum_{j=1}^{r}(-1)^{r-j}\left(\begin{array}{l}
r \\
j
\end{array}\right) j^{-n}(j t)^{r+n}\right) d \mu_{\xi}(t)\right| \\
& =\left|\left(\sum_{j=0}^{r}(-1)^{r-j}\left(\begin{array}{l}
r \\
j
\end{array}\right) j^{r}\right)\left(\int_{-\infty}^{\infty} t^{r+n} d \mu_{\xi}(t)\right)\right| \\
& =\left|\left(\Delta_{1}^{r} x^{r}\right)(0) \int_{-\infty}^{\infty} t^{r+n} d \mu_{\xi}(t)\right| \\
& =\left|r ! \int_{-\infty}^{\infty} t^{r+n} d \mu_{\xi}(t)\right| \\
& =r ! \int_{-\infty}^{\infty} t^{r+n} d \mu_{\xi}(t) \text {. }
\end{aligned}
$$

I.e. we have established

$$
\left|\Theta_{r, \xi}(f ; 0)\right|=r ! \int_{-\infty}^{\infty} t^{r+n} d \mu_{\xi}(t) .
$$

Thus by (24) and (25) we have established the claim of the theorem.

Corollary 4. Inequality (22) is sharp, that is attained at $\mathrm{x}=0$ by $\mathrm{f}(\mathrm{x})=\mathrm{x}^{\mathrm{r}}, \mathrm{r}$ even.

Proof. Notice that $\Delta_{t}^{r} x^{r}=r ! t^{r}$ and $\omega_{r}\left(f^{(n)}, t\right)=r ! t^{r}, t>0$. Thus

$$
\text { R.H.S. (22) }=r ! \int_{-\infty}^{\infty} t^{r} d \mu_{\xi}(t) \text {. }
$$


Also $f(0)=0$. Therefore

$$
\begin{aligned}
\text { L.H.S. 222 } & =\left|\Theta_{r, \xi}(f ; 0)\right| \\
& =\left|\int_{-\infty}^{\infty}\left(\sum_{j=1}^{r} \alpha_{j} j^{r} t^{r}\right) d \mu_{\xi}(t)\right| \\
& =\left|\int_{-\infty}^{\infty}\left(\sum_{j=0}^{r}(-1)^{r-j}\left(\begin{array}{l}
r \\
j
\end{array}\right) j^{r}\right) t^{r} d \mu_{\xi}(t)\right| \\
& =\left|\left(\Delta_{1}^{r} x^{r}\right)(0) \int_{-\infty}^{\infty} t^{r} d \mu_{\xi}(t)\right| \\
& =r ! \int_{-\infty}^{\infty} t^{r} d \mu_{\xi}(t),
\end{aligned}
$$

proving the claim.

Remark 5. On inequalities (10) and (22). We have the uniform estimates

$$
\left\|\Theta_{r, \xi}(f ; x)-f(x)-\sum_{k=1}^{n} \frac{f^{(k)}(x)}{k !} \delta_{k} c_{k, \xi}\right\|_{\infty, x} \leq \int_{-\infty}^{\infty} G_{n}(t) d \mu_{\xi}(t), n \in \mathbb{N},
$$

and

$$
\left\|\Theta_{r, \xi}(f ; x)-f(x)\right\|_{\infty, x} \leq \int_{-\infty}^{\infty} \omega_{r}(f,|t|) d \mu_{\xi}(t), \quad n=0 .
$$

Remark 6. The following regards the convergence of operators $\Theta_{r, \xi}$. From (8) we have

$$
G_{n}(t) \leq \frac{|t|^{n}}{n !} \omega_{r}\left(f^{(n)},|t|\right)
$$

Therefore by (26) we get

$$
\left\|\Theta_{r, \xi}(f ; x)-f(x)-\sum_{k=1}^{n} \frac{f^{(k)}(x)}{k !} \delta_{k} c_{k, \xi}\right\|_{\infty, x} \leq \frac{1}{n !} \int_{-\infty}^{\infty}|t|^{n} \omega_{r}\left(f^{(n)},|t|\right) d \mu_{\xi}(t) .
$$

Next using $\omega_{r}(f, \lambda t) \leq(\lambda+1)^{r} \omega_{r}(f, t), \lambda, t>0$, we get

$$
\begin{aligned}
\frac{1}{n !} \int_{-\infty}^{\infty}|t|^{n} \omega_{r}\left(f^{(n)},|t|\right) d \mu_{\xi}(t) & =\frac{1}{n !} \int_{-\infty}^{\infty}|t|^{n} \omega_{r}\left(f^{(n)}, \xi \cdot \frac{|t|}{\xi}\right) d \mu_{\xi}(t) \\
& \leq \frac{\omega_{r}\left(f^{(n)}, \xi\right)}{n !} \int_{-\infty}^{\infty}|t|^{n}\left(1+\frac{|t|}{\xi}\right)^{r} d \mu_{\xi}(t) .
\end{aligned}
$$

So we have proved that

$$
\begin{gathered}
K_{1}:=\left\|\Theta_{r, \xi}(f ; x)-f(x)-\sum_{k=1}^{n} \frac{f^{(k)}(x)}{k !} \delta_{k} c_{k, \xi}\right\|_{\infty, x} \\
\leq \frac{\omega_{r}\left(f^{(n)}, \xi\right)}{n !} \int_{-\infty}^{\infty}|t|^{n}\left(1+\frac{|t|}{\xi}\right)^{r} d \mu_{\xi}(t) .
\end{gathered}
$$


Similarly we get

$$
\begin{aligned}
\int_{-\infty}^{\infty} \omega_{r}(f,|t|) d \mu_{\xi}(t) & =\int_{-\infty}^{\infty} \omega_{r}\left(f, \xi \cdot \frac{|t|}{\xi}\right) d \mu_{\xi}(t) \\
& \leq \omega_{r}(f, \xi) \int_{-\infty}^{\infty}\left(1+\frac{|t|}{\xi}\right)^{r} d \mu_{\xi}(t)
\end{aligned}
$$

So that we have

$$
\begin{aligned}
K_{2}: & =\left\|\Theta_{r, \xi}(f ; x)-f(x)\right\|_{\infty} \\
\leq & \omega_{r}(f, \xi) \int_{-\infty}^{\infty}\left(1+\frac{|t|}{\xi}\right)^{r} d \mu_{\xi}(t) .
\end{aligned}
$$

In case that

$$
\int_{-\infty}^{\infty}|t|^{n}\left(1+\frac{|t|}{\xi}\right)^{r} d \mu_{\xi}(t) \leq \lambda_{1}
$$

$\forall \xi>0$, we get that $K_{1} \rightarrow 0$, as $\xi \rightarrow 0$.

In case that

$$
\int_{-\infty}^{\infty}\left(1+\frac{|t|}{\xi}\right)^{r} \mathrm{~d} \mu_{\xi}(t) \leq \lambda_{2}
$$

$\forall \xi>0$, we get that $K_{2} \rightarrow 0$, that is $\Theta_{r, \xi} \rightarrow \mathrm{I}$ (unit operator) uniformly, as $\xi \rightarrow 0$.

Note 7. The operators $\Theta_{r, \xi}$ are not in general positive and they are of convolution type.

Let $r=2, n=3$. Then $\alpha_{0}=\frac{23}{8}, \alpha_{1}=-2, \alpha_{2}=\frac{1}{8}$. Consider $f(t)=t^{2} \geq 0$ and $x=0$. Then

$$
\Theta_{2, \xi}\left(t^{2} ; 0\right)=-1.5 \int_{-\infty}^{\infty} t^{2} d \mu_{\xi}(t)<0
$$

where we assumed that $\int_{-\infty}^{\infty} t^{2} d \mu_{\xi}(t)<\infty$.

Remark 8. From [5] we get that

$$
\begin{aligned}
G_{n}(t) \leq & \frac{\omega_{r}\left(f^{(n)}, \xi\right)}{\xi^{r}}\left\{\sum_{k=0}^{n-1} \frac{(-1)^{k}}{k !(n-k-1) !(k+r+1)}\right. \\
& \left.\cdot\left[(\xi+|t|)^{n+r}-\xi^{r+k+1}(\xi+|t|)^{n-k-1}\right]\right\},
\end{aligned}
$$

for $\xi>0, \forall t \in \mathbb{R}$.

So by (26) we obtain

$$
\begin{aligned}
& \left\|\Theta_{r, \xi}(f ; x)-f(x)-\sum_{k=1}^{n} \frac{f^{(k)}(x)}{k !} \delta_{k} c_{k, \xi}\right\|_{\infty, x} \\
\leq & \frac{\omega_{r}\left(f^{(n)}, \xi\right)}{\xi^{r}}\left\{\sum_{k=0}^{n-1} \frac{(-1)^{k}}{k !(n-k-1) !(k+r+1)}\right. \\
& \left.\cdot\left[\int_{-\infty}^{\infty}(\xi+|t|)^{n+r} d \mu_{\xi}(t)-\xi^{r+k+1} \int_{-\infty}^{\infty}(\xi+|t|)^{n-k-1} d \mu_{\xi}(t)\right]\right\} .
\end{aligned}
$$


So from Remarks 5, 6 we derive

Theorem 9. Let $f \in C^{n}(\mathbb{R}), n \in \mathbb{Z}^{+}$. Set $c_{k, \xi}:=\int_{-\infty}^{\infty} t^{k} d \mu_{\xi}(t), k=1, \ldots, n$. Assume also $\omega_{\mathrm{r}}\left(\mathrm{f}^{(\mathrm{n})}, \mathrm{h}\right)<\infty, \forall \mathrm{h}>0$. It is also supposed that

$$
\int_{-\infty}^{\infty}|t|^{n}\left(1+\frac{|t|}{\xi}\right)^{r} d \mu_{\xi}(t)<\infty
$$

Then

$$
\begin{aligned}
& \left\|\Theta_{r, \xi}(f ; x)-f(x)-\sum_{k=1}^{n} \frac{f^{(k)}(x)}{k !} \delta_{k} c_{k, \xi}\right\|_{\infty, x} \\
\leq & \frac{\omega_{r}\left(f^{(n)}, \xi\right)}{n !} \int_{-\infty}^{\infty}|t|^{n}\left(1+\frac{|t|}{\xi}\right)^{r} d \mu_{\xi}(t) .
\end{aligned}
$$

When $n=0$ the sum in L.H.S. (34) collapses.

\section{Applications to General Trigonometric Singular Opera- tors}

We make

Remark 10. We need the following preliminary result.

Let $p$ and $m$ be integers with $1 \leq p \leq m$. We define the integral

$$
\mathrm{I}(\mathrm{m} ; \mathrm{p}):=\int_{-\infty}^{\infty} \frac{(\sin x)^{2 m}}{x^{2 p}} \mathrm{~d} x=2 \int_{0}^{\infty} \frac{(\sin x)^{2 m}}{x^{2 p}} \mathrm{~d} x .
$$

This is an (absolutely) convergent integral.

According to [11, page 210, item 1033, we obtain

$$
I(m ; p)=\pi \frac{(-1)^{p}(2 m) !}{4^{m-p}(2 p-1) !} \sum_{k=1}^{m}(-1)^{k} \frac{k^{2 p-1}}{(m-k) !(m+k) !} .
$$

In particular, for $p=m$ the above formula becomes

$$
\int_{0}^{\infty} \frac{(\sin x)^{2 m}}{x^{2 m}} d x=\pi(-1)^{m} m \sum_{k=1}^{m}(-1)^{k} \frac{k^{2 m-1}}{(m-k) !(m+k) !} .
$$

In this section we apply the general theory of this article to the trigonometric smooth general singular integral operators $\mathrm{T}_{\mathrm{r}, \xi}(\mathrm{f}, \mathrm{x})$ defined as follows. Let $\xi>0$. 
Let $f: \mathbb{R} \rightarrow \mathbb{R}$ be Borel measurable, we define for $x \in \mathbb{R}$ and $\beta \in \mathbb{N}$, the integral

$$
T_{r, \xi}(f ; x):=\frac{1}{W} \int_{-\infty}^{\infty}\left(\sum_{j=0}^{r} \alpha_{j} f(x+j t)\right)\left(\frac{\sin (t / \xi)}{t}\right)^{2 \beta} d t
$$

where

$$
\begin{aligned}
W & =\int_{-\infty}^{\infty}\left(\frac{\sin (t / \xi)}{t}\right)^{2 \beta} d t \\
& =2 \xi^{1-2 \beta} \int_{0}^{\infty}\left(\frac{\sin t}{t}\right)^{2 \beta} d t \\
& \stackrel{37}{=} 2 \xi^{1-2 \beta} \pi(-1)^{\beta} \beta \sum_{k=1}^{\beta}(-1)^{k} \frac{k^{2 \beta-1}}{(\beta-k) !(\beta+k) !} .
\end{aligned}
$$

We suppose that $T_{r, \xi}(f ; x) \in \mathbb{R}$ for all $x \in \mathbb{R}$.

We present our first result of this section

Theorem 11. Let $1 \leq n \leq 2 \beta-2$ and $k=1, \ldots, n$. The integrals

$$
\begin{aligned}
c_{k, \xi}: & =\frac{1}{W} \int_{-\infty}^{\infty} t^{k}\left(\frac{\sin (t / \xi)}{t}\right)^{2 \beta} d t \\
= & \left\{\begin{array}{ll}
0, & \text { for } k \text { odd } \\
\frac{\xi^{k}(-1)^{\frac{k}{2}}(2 \beta-1) !}{2^{k}(2 \beta-k-1) !} \frac{\sum_{j=1}^{\beta}(-1)^{j} \frac{j^{2 \beta-k-1}}{(\beta-j) !(\beta+j) !}}{\sum_{j=1}^{\beta}(-1)^{j} \frac{j^{2 \beta}-1}{(\beta-j) !(\beta+j) !}}, & \text { for } k \text { even }
\end{array},\right.
\end{aligned}
$$

are finite. Moreover, it holds

$$
\left|T_{r, \xi}(f ; x)-f(x)-\sum_{k=1}^{\lfloor n / 2\rfloor} \frac{f^{(2 k)}(x)}{(2 k) !} \delta_{2 k} c_{2 k, \xi}\right| \leq \frac{1}{W} \int_{-\infty}^{\infty} G_{n}(t)\left(\frac{\sin (t / \xi)}{t}\right)^{2 \beta} d t .
$$

When $n=1$ the sum in the L.H.S.401 colapses.

\section{Proof.}

We used Theorem 1 and relations (36) and (39).

Corollary 12. Assume $\omega_{\mathrm{r}}(\mathrm{f}, \xi)<\infty, \xi>0$. Then it holds for $\mathrm{n}=0$ and $\beta>1$ that

$$
\left|T_{r, \xi}(f ; x)-f(x)\right| \leq \frac{2}{W} \int_{0}^{\infty} \omega_{r}(f, t)\left(\frac{\sin (t / \xi)}{t}\right)^{2 \beta} d t .
$$

Proof. We are applying Corollary 2 here.

Note 13. The operators $\mathrm{T}_{\mathrm{r}, \xi}$ are not in general positive and they are of convolution type. 
Let $r=2, n=3$, and $\beta \geq 2$. Then $\alpha_{0}=\frac{23}{8}, \alpha_{1}=-2, \alpha_{2}=\frac{1}{8}$. Consider $f(t)=t^{2} \geq 0$ and $x=0$. Then

$$
\mathrm{T}_{2, \xi}\left(\mathrm{t}^{2} ; 0\right)=-1.5 \frac{1}{W} \int_{-\infty}^{\infty} \mathrm{t}^{2}\left(\frac{\sin (\mathrm{t} / \xi)}{\mathrm{t}}\right)^{2 \beta} \mathrm{dt}<0
$$

since

$$
\begin{aligned}
\frac{1}{W} \int_{-\infty}^{\infty} t^{2}\left(\frac{\sin (t / \xi)}{t}\right)^{2 \beta} d t & =\frac{\xi^{2}(-1)(2 \beta-1)(\beta-1)}{2} \frac{\sum_{j=1}^{\beta}(-1)^{j} \frac{j^{2 \beta-3}}{(\beta-j) !(\beta+j) !}}{\sum_{j=1}^{\beta}(-1)^{j} \frac{j^{2} \beta-1}{(\beta-j) !(\beta+j) !}} \\
& <\infty,
\end{aligned}
$$

by Theorem 11 .

Theorem 14. Let $f \in C^{n}(\mathbb{R}), n \in \mathbb{Z}^{+}$, and $\beta \geq 1+\left\lfloor\frac{\mathfrak{n}+\mathrm{r}+1}{2}\right\rfloor$. Assume also $\omega_{r}\left(f^{(\mathfrak{n})}, h\right)<\infty, \forall h>$ 0 . Then

$$
\begin{gathered}
\left\|T_{r, \xi}(f ; x)-f(x)-\sum_{k=1}^{\lfloor n / 2\rfloor} \frac{f^{(2 k)}(x)}{(2 k) !} \delta_{2 k} c_{2 k, \xi}\right\|_{\infty, x} \leq \frac{\xi^{n}}{n !} \frac{\omega_{r}\left(f^{(n)}, \xi\right)}{\pi(-1)^{\beta} \beta\left[\sum_{k=1}^{\beta}(-1)^{k} \frac{k^{2 \beta-1}}{(\beta-k) !(\beta+k) !}\right]} \\
\cdot\left[\int_{0}^{\infty} t^{n}(1+t)^{r}\left(\frac{\sin t}{t}\right)^{2 \beta} d t\right] .
\end{gathered}
$$

When $n=0,1$ the sum in L.H.S.(42) collapses.

Proof. For $i=0, \ldots, r$ we have that

$$
\begin{aligned}
\int_{0}^{\infty} t^{n+i}\left(\frac{\sin t}{t}\right)^{2 \beta} d t & =\frac{\pi(-1)^{\beta-\frac{n+i}{2}}(2 \beta) !}{2^{n+i+1}[2 \beta-n-i-1] !} \sum_{k=1}^{\beta}(-1)^{k} \frac{k^{2 \beta-n-i-1}}{(\beta-k) !(\beta+k) !} \\
& <\infty
\end{aligned}
$$

in the case $n+i$ even (because $\beta \geq 1+\left\lfloor\frac{\mathfrak{n}+r+1}{2}\right\rfloor \geq 1+\frac{\mathfrak{n}+i}{2}$ ). 
Furthermore, when $n+i$ is odd we get

$$
\begin{aligned}
\int_{0}^{\infty} t^{n+i}\left(\frac{\sin t}{t}\right)^{2 \beta} d t & =\int_{0}^{1} t^{n+i}\left(\frac{\sin t}{t}\right)^{2 \beta} d t+\int_{1}^{\infty} t^{n+i}\left(\frac{\sin t}{t}\right)^{2 \beta} d t \\
& \leq \int_{0}^{1} t^{n+i-1}\left(\frac{\sin t}{t}\right)^{2 \beta} d t+\int_{1}^{\infty} t^{n+i+1}\left(\frac{\sin t}{t}\right)^{2 \beta} d t \\
& \leq \int_{0}^{\infty} t^{n+i-1}\left(\frac{\sin t}{t}\right)^{2 \beta} d t+\int_{0}^{\infty} t^{n+i+1}\left(\frac{\sin t}{t}\right)^{2 \beta} d t \\
& =\frac{\pi(-1)^{\beta-\frac{n+i-1}{2}}(2 \beta) !}{2^{n+i}[2 \beta-n-i] !} \sum_{k=1}^{\beta}(-1)^{k} \frac{k^{2 \beta-n-i}}{(\beta-k) !(\beta+k) !} \\
& +\frac{\pi(-1)^{\beta-\frac{n+i+1}{2}}(2 \beta) !}{2^{n+i+2}[2 \beta-n-i-2] !} \sum_{k=1}^{\beta}(-1)^{k} \frac{k^{2 \beta-n-i-2}}{(\beta-k) !(\beta+k) !} \\
< & \infty,
\end{aligned}
$$

by $\beta \geq 1+\left\lfloor\frac{n+r+1}{2}\right\rfloor \geq 1+\frac{n+i+1}{2}$.

Therefore it holds

$$
\int_{0}^{\infty} \sum_{i=0}^{r}\left(\begin{array}{l}
r \\
i
\end{array}\right) t^{n+i}\left(\frac{\sin t}{t}\right)^{2 \beta} d t=\sum_{i=0}^{r}\left(\begin{array}{l}
r \\
i
\end{array}\right) \int_{0}^{\infty} t^{n+i}\left(\frac{\sin t}{t}\right)^{2 \beta} d t<\infty .
$$

Hence

$$
\begin{gathered}
\frac{1}{W} \int_{-\infty}^{\infty}|t|^{n}\left(1+\frac{|t|}{\xi}\right)^{r}\left(\frac{\sin (t / \xi)}{t}\right)^{2 \beta} d t=\frac{2}{W} \int_{0}^{\infty} t^{n}\left(1+\frac{t}{\xi}\right)^{r}\left(\frac{\sin (t / \xi)}{t}\right)^{2 \beta} d t \\
=\frac{2 \xi^{n-2 \beta+1}}{W} \int_{0}^{\infty} t^{n}(1+t)^{r}\left(\frac{\sin t}{t}\right)^{2 \beta} d t \\
\stackrel{39}{=} \frac{\xi^{n}}{\pi \beta\left[(-1)^{\beta} \sum_{k=1}^{\beta}(-1)^{k} \frac{k^{2 \beta-1}}{(\beta-k) !(\beta+k) !}\right]} \int_{0}^{\infty} t^{n}(1+t)^{r}\left(\frac{\sin t}{t}\right)^{2 \beta} d t \\
=\frac{\xi^{n}}{\pi \beta\left[(-1)^{\beta} \sum_{k=1}^{\beta}(-1)^{k} \frac{k^{2 \beta-1}}{(\beta-k) !(\beta+k) !}\right]} \int_{0}^{\infty} \sum_{i=0}^{r}\left(\begin{array}{l}
r \\
i
\end{array}\right) t^{n+i}\left(\frac{\sin t}{t}\right)^{2 \beta} d t \\
<\infty,
\end{gathered}
$$

which implies that

$$
\frac{1}{W} \int_{-\infty}^{\infty}|t|^{n}\left(1+\frac{|t|}{\xi}\right)^{r}\left(\frac{\sin (t / \xi)}{t}\right)^{2 \beta} d t<\infty
$$

Applying Theorem 9 here we obtain the inequality (42). 


\section{Applications to Particular Trigonometric Singular Oper- ators}

In this section we work on the approximation results given in the previous section, for some particular values of $n$ and $\beta$.

\section{Case $\beta=1$.}

We have the following value corresponding to formula (39)

$$
W=\pi \xi^{-1} .
$$

None of the previous results hold in this case.

Case $\beta=2$.

We have the following value corresponding to formula (39)

$$
W=\frac{2 \pi}{3} \xi^{-3}
$$

Theorem 15. It holds, for $\mathrm{n}=1$

$$
\left|T_{r, \xi}(f ; x)-f(x)\right| \leq \frac{3}{2 \pi} \xi^{3} \int_{-\infty}^{\infty} G_{1}(t)\left(\frac{\sin (t / \xi)}{t}\right)^{4} d t
$$

and, for $\mathrm{n}=2$

$$
\left|T_{r, \xi}(f ; x)-f(x)-\frac{f^{\prime \prime}(x)}{2} \delta_{2} c_{2, \xi}\right| \leq \frac{3}{2 \pi} \xi^{3} \int_{-\infty}^{\infty} G_{2}(t)\left(\frac{\sin (t / \xi)}{t}\right)^{4} d t .
$$

Proof.

By Theorem 11, with $\beta=2, n=1,2$.

Corollary 16. Assume $\omega_{r}(f, \xi)<\infty, \xi>0$. Then it holds for $\mathrm{n}=0$ that

$$
\left|T_{r, \xi}(f ; x)-f(x)\right| \leq \frac{3}{\pi} \xi^{3} \int_{0}^{\infty} \omega_{r}(f, t)\left(\frac{\sin (t / \xi)}{t}\right)^{4} d t .
$$

Proof. By Corollary 12.

Theorem 17. Let $f \in C^{1}(\mathbb{R})$. Assume $\omega_{1}\left(f^{\prime}, h\right)<\infty, \forall h>0$. Then

$$
\left\|T_{1, \xi}(f)-f\right\|_{\infty} \leq \frac{3 \xi}{\pi}\left[\ln 2+\frac{\pi}{4}\right] \omega_{1}\left(f^{\prime}, \xi\right) .
$$


Proof. We are applying Theorem 14 here for $n=r=1$, etc.

Case $\beta=3$.

We have the following value corresponding to formula (39)

We have

$$
W=\frac{11 \pi}{20} \xi^{-5}
$$

\section{Theorem 18. It holds}

$$
\begin{gathered}
\left|T_{r, \xi}(f ; x)-f(x)\right| \leq \frac{20}{11 \pi} \xi^{5} \int_{-\infty}^{\infty} G_{1}(t)\left(\frac{\sin (t / \xi)}{t}\right)^{6} d t . \\
\left|T_{r, \xi}(f ; x)-f(x)-\frac{f^{\prime \prime}(x)}{2} \delta_{2} c_{2, \xi}\right| \leq \frac{20}{11 \pi} \xi^{5} \int_{-\infty}^{\infty} G_{2}(t)\left(\frac{\sin (t / \xi)}{t}\right)^{6} d t . \\
\left|T_{r, \xi}(f ; x)-f(x)-\frac{f^{\prime \prime}(x)}{2} \delta_{2} c_{2, \xi}\right| \leq \frac{20}{11 \pi} \xi^{5} \int_{-\infty}^{\infty} G_{3}(t)\left(\frac{\sin (t / \xi)}{t}\right)^{6} d t . \\
\left|T_{r, \xi}(f ; x)-f(x)-\frac{f^{\prime \prime}(x)}{2} \delta_{2} c_{2, \xi}-\frac{f^{(4)}(x)}{24} \delta_{4} c_{4, \xi}\right| \leq \frac{20}{11 \pi} \xi^{5} \int_{-\infty}^{\infty} G_{4}(t)\left(\frac{\sin (t / \xi)}{t}\right)^{6} d t .
\end{gathered}
$$

Proof.

By Theorem 11, for $\beta=3$, and $n=1,2,3,4$.

Corollary 19. Assume $\omega_{\mathrm{r}}(\mathrm{f}, \xi)<\infty, \xi>0$. Then it holds for $\mathrm{n}=0$ that

$$
\left|T_{r, \xi}(f ; x)-f(x)\right| \leq \frac{40}{11 \pi} \xi^{5} \int_{0}^{\infty} \omega_{r}(f, t)\left(\frac{\sin (t / \xi)}{t}\right)^{6} d t
$$

Proof. We are applying Corollary 12 here.

Theorem 20. Let $f \in C^{n}(\mathbb{R}), n \in \mathbb{Z}^{+}$, and $n+r=1,2,3,4$. Assume also $\omega_{r}\left(f^{(n)}, h\right)<\infty, \forall h>0$. Then

$$
\begin{aligned}
& \left\|T_{r, \xi}(f ; x)-f(x)-\sum_{k=1}^{\lfloor n / 2\rfloor} \frac{f^{(2 k)}(x)}{(2 k) !} \delta_{2 k} c_{2 k, \xi}\right\|_{\infty, x} \\
& \leq \frac{40}{11 \pi}\left[\int_{0}^{\infty} t^{n}(1+t)^{r}\left(\frac{\sin t}{t}\right)^{6} d t\right] \frac{\omega_{r}\left(f^{(n)}, \xi\right) \xi^{n}}{n !} .
\end{aligned}
$$

When $n=0,1$ the sum in L.H.S. (52) collapses. 
Proof. We are applying Theorem 14 here.

Case $\beta=4$.

We have the following value corresponding to formula (39)

$$
W=\frac{151 \pi}{315} \xi^{-7}
$$

Theorem 21. It holds

$$
\begin{gathered}
\left|T_{r, \xi}(f ; x)-f(x)\right| \leq \frac{315}{151 \pi} \xi^{7} \int_{-\infty}^{\infty} G_{1}(t)\left(\frac{\sin (t / \xi)}{t}\right)^{8} d t . \\
\left|T_{r, \xi}(f ; x)-f(x)-\frac{f^{\prime \prime}(x)}{2} \delta_{2} c_{2, \xi}\right| \leq \frac{315}{151 \pi} \xi^{7} \int_{-\infty}^{\infty} G_{2}(t)\left(\frac{\sin (t / \xi)}{t}\right)^{8} d t . \\
\left|T_{r, \xi}(f ; x)-f(x)-\frac{f^{\prime \prime}(x)}{2} \delta_{2} c_{2, \xi}\right| \leq \frac{315}{151 \pi} \xi^{7} \int_{-\infty}^{\infty} G_{3}(t)\left(\frac{\sin (t / \xi)}{t}\right)^{8} d t . \\
\leq \frac{315}{151 \pi} \xi^{7} \int_{-\infty}^{\infty} G_{6}(t)\left(\frac{\sin (t / \xi)}{t}\right)^{8} d t .
\end{gathered}
$$

Proof.

We used Theorem 11 , with $\beta=4$, and $n=1,2,3,6$.

Corollary 22. Assume $\omega_{\mathrm{r}}(\mathrm{f}, \xi)<\infty, \xi>0$. Then for $\mathrm{n}=0$ it holds

$$
\left|T_{r, \xi}(f ; x)-f(x)\right| \leq \frac{630}{151 \pi} \xi^{7} \int_{0}^{\infty} \omega_{r}(f, t)\left(\frac{\sin (t / \xi)}{t}\right)^{8} d t .
$$

Proof. We are applying Corollary 12 here.

Theorem 23. Let $f \in C^{n}(\mathbb{R}), n \in \mathbb{Z}^{+}$, and $n+r=1,2,3,4,5,6$. Assume also $\omega_{r}\left(f^{(n)}, h\right)<$ $\infty, \forall \mathrm{h}>0$. Then

$$
\begin{gathered}
\left\|T_{r, \xi}(f ; x)-f(x)-\sum_{k=1}^{\lfloor n / 2\rfloor} \frac{f^{(2 k)}(x)}{(2 k) !} \delta_{2 k} c_{2 k, \xi}\right\|_{\infty, x} \\
\leq \frac{630}{151 \pi}\left[\int_{0}^{\infty} t^{n}(1+t)^{r}\left(\frac{\sin t}{t}\right)^{8} d t\right] \frac{\omega_{r}\left(f^{(n)}, \xi\right) \xi^{n}}{n !} .
\end{gathered}
$$


When $n=0,1$ the sum in L.H.S.(58) collapses.

Proof. We are applying Theorem 14 here.

Case $\beta=6$.

We have the following value corresponding to formula (39)

$$
W=\int_{-\infty}^{\infty}\left(\frac{\sin (t / \xi)}{t}\right)^{12} d t=\frac{655177 \pi}{1663200} \xi^{-11}
$$

Theorem 24. It holds

$$
\begin{gathered}
\left|T_{r, \xi}(f ; x)-f(x)\right| \leq \frac{1663200}{655177 \pi} \xi^{11} \int_{-\infty}^{\infty} G_{1}(t)\left(\frac{\sin (t / \xi)}{t}\right)^{12} d t . \\
\left|T_{r, \xi}(f ; x)-f(x)-\frac{f^{\prime \prime}(x)}{2} \delta_{2} c_{2, \xi}\right| \leq \frac{1663200}{655177 \pi} \xi^{11} \int_{-\infty}^{\infty} G_{2}(t)\left(\frac{\sin (t / \xi)}{t}\right)^{12} d t . \\
\left|T_{r, \xi}(f ; x)-f(x)-\frac{f^{\prime \prime}(x)}{2} \delta_{2} c_{2, \xi}\right| \leq \frac{1663200}{655177 \pi} \xi^{11} \int_{-\infty}^{\infty} G_{3}(t)\left(\frac{\sin (t / \xi)}{t}\right)^{12} d t . \\
\leq \mid \frac{1663200}{655177 \pi} \xi^{11} \int_{-\infty}^{\infty} G_{6}(t)\left(\frac{\sin (t / \xi)}{t}\right)^{12} d t . \\
\left|T_{r, \xi}(f ; x)-f(x)-\frac{f^{\prime \prime}(x)}{2} \delta_{2} c_{2, \xi}-\frac{f^{(4)}(x)}{24} \delta_{4} c_{4, \xi}-\frac{f^{(6)}(x)}{720} \delta_{6} c_{6, \xi}\right| \\
f(x)-\frac{f^{\prime \prime}(x)}{2} \delta_{2} c_{2, \xi}-\frac{f^{(4)}(x)}{24} \delta_{4} c_{4, \xi}-\frac{f^{(6)}(x)}{720} \delta_{6} c_{6, \xi}-\frac{f^{(8)}(x)}{8 !} \delta_{8} c_{8, \xi}-\frac{f^{(10)}(x)}{10 !} \delta_{10} c_{10, \xi} \mid \\
\leq \frac{1663200}{655177 \pi} \xi^{11} \int_{-\infty}^{\infty} G_{10}(t)\left(\frac{\sin (t / \xi)}{t}\right)^{12} d t .
\end{gathered}
$$

\section{Proof.}

We used Theorem 11, with $\beta=6$, and $n=1,2,3,6,10$.

Corollary 25. Assume $\omega_{\mathrm{r}}(\mathrm{f}, \xi)<\infty, \xi>0$. Then for $\mathrm{n}=0$ it holds

$$
\left|T_{r, \xi}(f ; x)-f(x)\right| \leq \frac{3326400}{655177 \pi} \xi^{11} \int_{0}^{\infty} \omega_{r}(f, t)\left(\frac{\sin (t / \xi)}{t}\right)^{12} d t .
$$

Proof. We are applying Corollary 12 here. 
Theorem 26. Let $f \in C^{n}(\mathbb{R}), n \in \mathbb{Z}^{+}$, and $n+r \in\{1,2,3, \ldots, 10\}$. Assume also $\omega_{r}\left(f^{(n)}, h\right)<$ $\infty, \forall \mathrm{h}>0$. Then

$$
\begin{gathered}
\left\|T_{r, \xi}(f ; x)-f(x)-\sum_{k=1}^{\lfloor n / 2\rfloor} \frac{f^{(2 k)}(x)}{(2 k) !} \delta_{2 k} c_{2 k, \xi}\right\|_{\infty, x} \leq \frac{3326400}{655177 \pi} \\
\cdot\left[\int_{0}^{\infty} t^{n}(1+t)^{r}\left(\frac{\sin t}{t}\right)^{12} d t\right] \frac{\omega_{r}\left(f^{(n)}, \xi\right) \xi^{n}}{n !} .
\end{gathered}
$$

When $\mathrm{n}=0,1$ the sum in L.H.S.(46) collapses.

Proof. We are applying Theorem 14 here.

Case $\beta=10$.

We have the following value corresponding to formula (39)

$$
W=\int_{-\infty}^{\infty}\left(\frac{\sin (t / \xi)}{t}\right)^{20} d t=\frac{37307713155613 \pi}{121645100408832} \xi^{-19} .
$$

Theorem 27. It holds

$$
\begin{gathered}
\left|T_{r, \xi}(f ; x)-f(x)\right| \leq \frac{121645100408832}{37307713155613 \pi} \xi^{19} \\
\cdot \int_{-\infty}^{\infty} G_{1}(t)\left(\frac{\sin (t / \xi)}{t}\right)^{20} d t . \\
\left|T_{r, \xi}(f ; x)-f(x)-\frac{f^{\prime \prime}(x)}{2} \delta_{2} c_{2, \xi}\right| \leq \frac{121645100408832}{37307713155613 \pi} \xi^{19} \\
\mid \int_{-\infty}^{\infty} G_{2}(t)\left(\frac{\sin (t / \xi)}{t}\right)^{20} d t . \\
\left|T_{r, \xi}(f ; x)-f(x)-\frac{f^{\prime \prime}(x)}{2} \delta_{2} c_{2, \xi}\right| \leq \frac{121645100408832}{37307713155613 \pi} \xi^{19} \\
\leq \int_{-\infty}^{\infty} G_{3}(t)\left(\frac{\sin (t / \xi)}{t}\right)^{20} d t . \\
\frac{121645100408832}{37307713155613 \pi} \xi^{19} \int_{-\infty}^{\infty} G_{6}(t)\left(\frac{\sin (t / \xi)}{t}\right)^{20} d t . \\
\left|T_{r, \xi}(f ; x)-f(x)-\frac{f^{\prime \prime}(x)}{2} \delta_{2} c_{2, \xi}-\frac{f^{(4)}(x)}{24} \delta_{4} c_{4, \xi}-\frac{f^{(6)}(x)}{720} \delta_{6} c_{6, \xi}\right| \\
\leq
\end{gathered}
$$




$$
\begin{gathered}
\left|T_{r, \xi}(f ; x)-f(x)-\frac{f^{\prime \prime}(x)}{2} \delta_{2} c_{2, \xi}-\frac{f^{(4)}(x)}{24} \delta_{4} c_{4, \xi}-\frac{f^{(6)}(x)}{720} \delta_{6} c_{6, \xi}-\frac{f^{(8)}(x)}{8 !} \delta_{8} c_{8, \xi}-\frac{f^{(10)}(x)}{10 !} \delta_{10} c_{10, \xi}\right| \\
\leq \frac{121645100408832}{37307713155613 \pi} \xi^{19} \int_{-\infty}^{\infty} G_{10}(t)\left(\frac{\sin (t / \xi)}{t}\right)^{20} d t .
\end{gathered}
$$

\section{Proof.}

We used Theorem 11 , with $\beta=10$, and $n=1,2,3,6,10$.

Corollary 28. Assume $\omega_{\mathrm{r}}(\mathrm{f}, \xi)<\infty, \xi>0$. Then for $\mathrm{n}=0$ it holds

$$
\left|T_{r, \xi}(f ; x)-f(x)\right| \leq \frac{243290200817664}{37307713155613 \pi} \xi^{19} \int_{0}^{\infty} \omega_{r}(f, t)\left(\frac{\sin (t / \xi)}{t}\right)^{20} d t .
$$

Proof. We are applying Corollary 12 here.

Theorem 29. Let $f \in C^{n}(\mathbb{R}), n \in \mathbb{Z}^{+}$, and $n+r=\{1,2, \ldots, 18\}$. Assume also $\omega_{r}\left(f^{(n)}, h\right)<$ $\infty, \forall \mathrm{h}>0$. Then

$$
\begin{gathered}
\left\|T_{r, \xi}(f ; x)-f(x)-\sum_{k=1}^{\lfloor n / 2\rfloor} \frac{f^{(2 k)}(x)}{(2 k) !} \delta_{2 k} c_{2 k, \xi}\right\|_{\infty, x} \leq \frac{243290200817664}{37307713155613 \pi} \\
\cdot\left[\int_{0}^{\infty} t^{n}(1+t)^{r}\left(\frac{\sin t}{t}\right)^{20} d t\right] \frac{\omega_{r}\left(f^{(n)}, \xi\right) \xi^{n}}{n !} .
\end{gathered}
$$

When $n=0,1$ the sum in L.H.S.(73) collapses.

Proof. We are applying Theorem 14 here.

Acknowledgement. The authors would like to thank Professor V. Papanicolaou of National Technical University of Athens, Greece, for having fruitful discussions during the preparation of this article.

Received: February 2011. Accepted: April 2011.

\section{References}

[1] G.A. Anastassiou, Rate of convergence of non-positive linear convolution type operators. A sharp inequality, J. Math. Anal. and Appl., 142 (1989), 441-451.

[2] G.A. Anastassiou, Sharp inequalities for convolution type operators, Journal of Approximation Theory, 58 (1989), 259-266. 
[3] G.A. Anastassiou, Moments in Probability and Approximation Theory, Pitman Research Notes in Math., Vol. 287, Longman Sci. \& Tech., Harlow, U.K., 1993.

[4] G.A. Anastassiou, Quantitative Approximations, Chapman \& Hall/CRC, Boca Raton, New York, 2001.

[5] G.A. Anastassiou, Basic Convergence with Rates of Smooth Picard Singular Operators, $J$. Comput. Anal. Appl., 8 (2006), 313-334.

[6] G.A. Anastassiou and S. Gal, Convergence of generalized singular integrals to the unit, univariate case, Math. Inequalities $\&$ Applications, 3, No. 4 (2000), 511-518.

[7] G.A. Anastassiou and S. Gal, Convergence of generalized singular integrals to the unit, multivariate case, Applied Math. Rev., Vol. 1, World Sci. Publ. Co., Singapore, 2000, pp. 1-8.

[8] George A. Anastassiou, Razvan A. Mezei, Uniform Convergence with Rates of Smooth GaussWeierstrass Singular Integral Operators, Applicable Analysis, 88:7 (2009), 1015 - 1037.

[9] G.A. Anastassiou and R. A. Mezei, Uniform Convergence with Rates of Smooth PoissonCauchy Type Singular Integral Operators, Mathematical and Computer Modelling 50 (2009), 1553-1570.

[10] R.A. DeVore and G.G. Lorentz, Constructive Approximation, Springer-Verlag, Vol. 303, Berlin, New York, 1993.

[11] Joseph Edwards, A treatise on the integral calculus, Vol II, Chelsea, New York, 1954.

[12] S.G. Gal, Remark on the degree of approximation of continuous functions by singular integrals, Math. Nachr., 164 (1993), 197-199.

[13] S.G. Gal, Degree of approximation of continuous functions by some singular integrals, Rev. Anal. Numér, Théor. Approx., (Cluj), Tome XXVII, No. 2 (1998), 251-261.

[14] J.B. Kemperman, The general moment problem, a geometric approach, Ann. Math. Stat., 39 (1968), 93-122.

[15] R.N. Mohapatra and R.S. Rodriguez, On the rate of convergence of singular integrals for Hölder continuous functions, Math. Nachr., 149 (1990), 117-124.

[16] L. Schumaker, Spline Functions. Basic Theory, J. Wiley \& Sons, New York, 1981. 